\title{
Gold Nanoparticles on MgO - A Revisit using Cs-Corrected STEM
}

\author{
Y. Han and Z.Y. Li
}

Nanoscale Physics Research Laboratory, School of Physics and Astronomy, University of Birmingham, Birmingham B15 2TT, UK

Previously, high-resolution transmission electron microscopy (HREM) has been commonly used for the purpose of understanding the 3D structures of $\mathrm{Au}$ nanoparticles [1,2]. However, the phase contrast images may only be interpretable with the help of simulations, with accurate structural models required for the first instance. This can be a real challenge in detecting low-coordination Au atoms in the nanoparticles, which has been reported to be the most important factor determining the catalytic activities [3]. In comparison, high-resolution aberration-corrected scanning transmission electron microscopy (AC-STEM) imaging has been applied successfully in the characterisation of metallic nanoparticles on various substrates [4,5]. The high-angle annular dark-field (HAADF) images taken under STEM mode exhibit an advantage of allowing us to see single atoms directly and analyzing the contrast quantitatively [4-6].

In this context, $\mathrm{Au}$ nanoparticles deposited on $\mathrm{MgO}$ smoke crystals at room temperature via thermal evaporation were characterised using a $200 \mathrm{kV}$ spherical aberration corrected JEM 2100f STEM equipped with a JEOL HAADF detector. Fig.1a is a low-magnification micrograph of the sample and Fig. $1 \mathrm{~b}$ is an atomic-resolution image showing one of the Au nanoclusters supported on one side surface of a $\mathrm{MgO}$. This cluster roughly adopted a half truncated octahedron shape with the electron beam aligned with the [001] zone axis of the $\mathrm{MgO}$. To obtain quantitative information about atomic arrangement of the cluster, high-frequency noises were first filtered using fast Fourier transform (FFT). The maximum intensities were then used to locate the positions of the atomic columns. The $\mathrm{MgO}$ substrate recorded in the same image was used to achieve an absolute scale. Both the in-layer (parallel to the interface) spacings and layer-to-layer spacings (vertical to the interface) were measured, with the average values plotted in Fig. 2. Here the atomic layers parallel to the $\mathrm{Au} / \mathrm{MgO}$ interface were numbered from the $\mathrm{Au} / \mathrm{MgO}$ interface. It can be seen that, away from the interface, the average lateral spacing firstly adopted $\mathrm{d}_{002}$ of $\mathrm{MgO}$ (layers 1,2). It then went down (layers 3-8) towards the $\mathrm{d}_{002}$ value of bulk $\mathrm{Au}$, and finally showed a strong relaxation (layers 9-11). Interestingly, the vertical spacing displayed an overall contraction as the layer moving away from the interface. This is the first direct evidence that the relaxation for the atoms in the top two layers (lateral) was strongly coupled with a vertical contraction for $\mathrm{Au}$ nanoparticles on $\mathrm{MgO}(100)$ surface. It is noted that out-of-plane contractions for surface atoms in Au nanoparticles of 3-5 nm in diameter on carbon support had been suggested by coherent diffraction [7]. The contraction estimated here is $0.17 \AA$, being comparable with that reported in reference [7] for the $\{001\}$ surface atoms, at $0.13 \AA$. The present study demonstrated that the latest advancement in AC-STEM could provide vast unprecedented opportunities to access direct atomic information at surfaces and interfaces. Combined with time evolving measurements, one should be able to study the dynamic behaviors of nanoparticles on a variety of supports in atomic details. 


\section{References}

[1] B. Pauwels, G. Van Tendeloo, W. Bouwen, L.T. Kuhn, P. Lievens, H. Lei, M. Hou, Phys. Rev. B 62 (2000) 10383.

[2] S. Giorgio, M. Cabie, C.R. Henry, Gold Bull. 41 (2008) 167.

[3] M. Haruta, Gold Bull. 37 (2004) 27.

[4] Z.Y. Li, N.P. Young, M. Di Vece, S. Palomba, R.E. Palmer, A.L. Bleloch, B.C. Curley, R.L. Johnston, J. Jiang, J. Yuan, Nature 451 (2008) 46.

[5] S. Van Aert, K.J. Batenburg, M.D. Rossell, R. Erni, G. Van Tendeloo, Nature 470 (2011) 374.

[6] Y. Liu, C.J. Jia, J. Yamasaki, O. Terasaki, F. Schüth, Angew. Chem. Int. Ed. 49 (2010) 5771.

[7] W.J. Huang, R. Sun, J. Tao, L.D. Menard, R.G. Nuzzo, J.M. Zuo, Nature Mater. 7 (2008) 308.

[8] The authors acknowledge the Engineering and Physical Sciences Research Council (UK) and the Birmingham Science City project (AWM) for support.

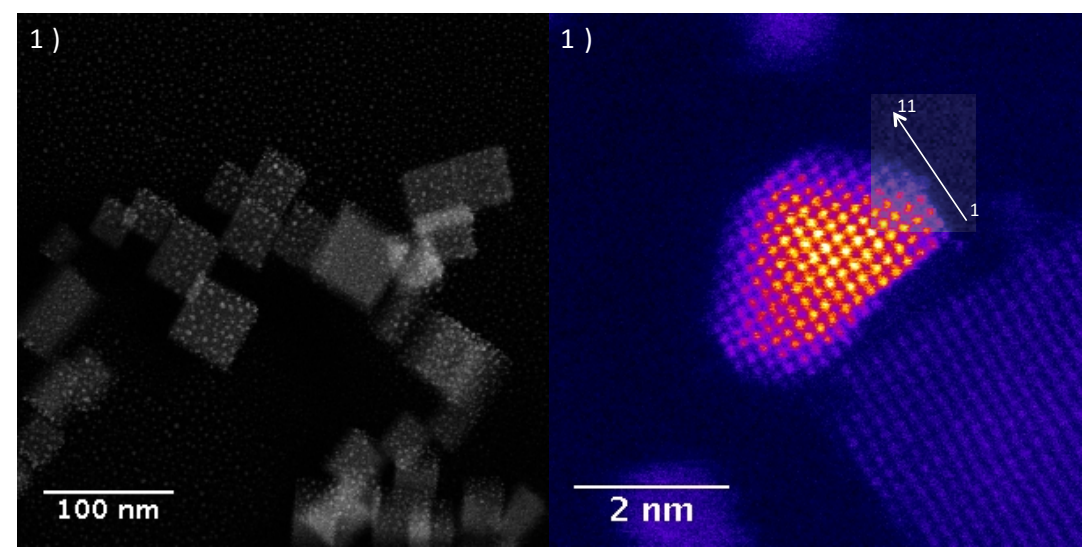

FIG. 2. (a) Overview of the Au nanoparticles on MgO crystals. (b) HAADF-STEM image of a Au nanoparticle supported on $\mathrm{MgO}$. The atomic layers parallel to the $\mathrm{Au} / \mathrm{MgO}$ interface were numbered from the $\mathrm{Au} / \mathrm{MgO}$ interface.

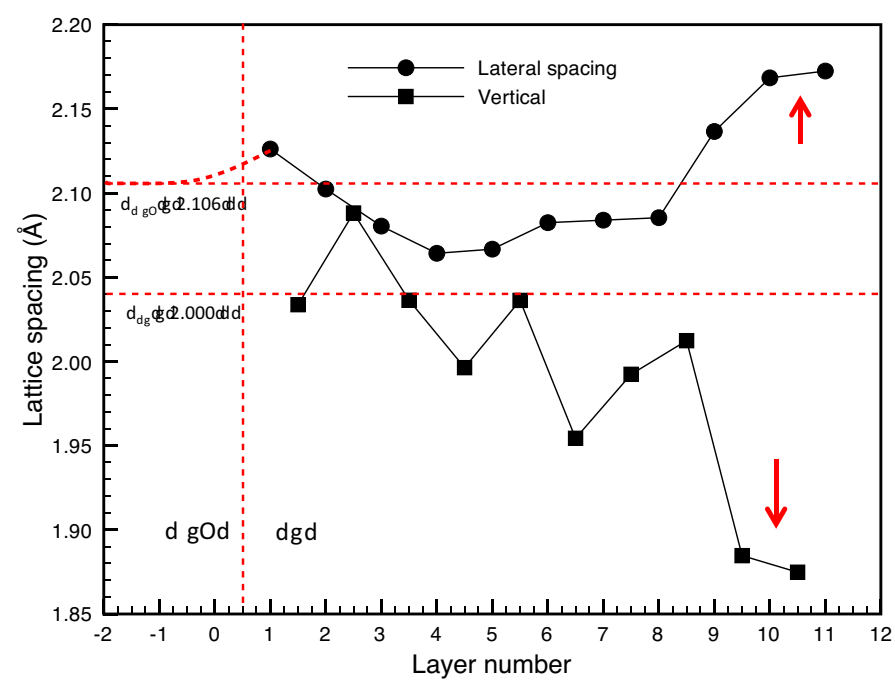

FIG. 3. The average lateral and vertical lattice spacings vs. layer number. The occurrence of lateral relaxation for the atoms in the top two layers coupled with a strong vertical contraction is indicated by the arrows. The half units in the $\mathrm{x}$-axis for the vertical values are used to demonstrate their relative positions with the lateral values. 